\title{
What should we know about the person behind a TTO?
}

\author{
Floortje van Nooten ${ }^{1} \cdot$ Jan Busschbach ${ }^{2} \cdot$ Michel van Agthoven ${ }^{3} \cdot$ Job van Exel $^{1} \cdot$ Werner Brouwer $^{1,4}$
}

Published online: 18 April 2018

(c) Springer-Verlag GmbH Germany, part of Springer Nature 2018

\section{Introduction}

The time trade-off (TTO) method is widely used to obtain quality of life scores for health states, but its validity is not uncontested, for instance because TTO values for similar health states can differ substantially between studies [1]. This could be caused by differences between studies in how the procedure is applied, but could also relate to differences in study samples.

In a TTO, respondents are asked to value health states by making trade-offs between length and quality of life within a limited remaining lifespan. This is a cognitively demanding task, which for some respondents may be more difficult to complete than for others. Moreover, despite the hypothetical nature of the exercise, having to imagine giving up life years from a finite amount of remaining life years to increase quality of life may invoke different emotions in respondents. This may be related to different expectations about their own remaining life expectancy or to different attitudes towards and experiences with this existentialistic question. These different emotional responses to the exercise may lead to answers that do not necessarily relate to the actual value of the health state presented to respondents, which TTO obviously hopes to elicit. We argue that to come to a better understanding of variations in responses to TTO questions, more systematic attention is required for the persons behind TTO responses.

Werner Brouwer

brouwer@eshpm.eur.nl

1 Erasmus School of Health Policy and Management, Erasmus University Rotterdam, Rotterdam, The Netherlands

2 Section Medical Psychology and Psychotherapy, Department of Psychiatry, Erasmus MC, Rotterdam, The Netherlands

3 Janssen-Cilag BV, Tilburg, The Netherlands

4 Institute for Medical Technology Assessment, Erasmus University Rotterdam, Rotterdam, The Netherlands

\section{TTO}

A TTO question typically asks respondents to imagine being in a particular imperfect health state for a certain period of time, say 10 years, after which they will die. Respondents are then asked to consider an alternative scenario in which they are in full health but live for a shorter period of time. Subsequently, respondents are requested to indicate how many years living in full health for them would be equivalent to living 10 years in the imperfect health state. This point of indifference can be used to compute a value for the imperfect health state relative to full health. To do so, the scale on which health utilities are measured, is usually normalized by setting the value of the state 'dead' equal to 0 and that of 'full health' equal to one. Then, if a respondent indicates, for example, that living for 10 years with moderate pain and severe problems with mobility (with value $\beta$ ) is equivalent to living 8.5 years in full health (with value one), the value of the imperfect health state $(\beta)$ for this respondent can be computed as follows. Ten years in $\beta$ equals 8.5 years in full health, can be written as $10 * \beta=8.5 * 1$ or $\beta=8.5 / 10=0.85$.

This can be formalised as follows:

$T * v(X)=P * v(\mathrm{FH})+(T-P) * v(D)$,

where $v(\mathrm{FH})$ denotes the value of full health, $v(X)$ the value of imperfect health state $X$ and $v(\mathrm{D})$ the value of being dead. A respondent is then asked to reveal the number of years $P$ (with $P<T$ ) in full health (after which death follows), that this person considers equal to $T$ years in the imperfect state $X$. Given that $v(\mathrm{FH})$ is set to one and $v(D)$ to 0 , Eq. (1) reduces to

$T * v(X)=P$.

Abstracting from issues such as discounting [2], for the sake of simplicity, the value of imperfect health state $X$ can then be computed by dividing the years in full health $(P)$ by the number of years in imperfect health $(T)$, thus:

$v(X)=\frac{P}{T}$. 
The remaining life years in full health $T$ presented to respondents varies between studies. Common time frames include 10 years or the subject's expected remaining life expectancy [3-5]. Equation (3) suggests that this variation in subject's expected remaining life expectancy does not affect the value of the health state, as long as people tradeoff life time proportionally for different periods of time [6]. Although this makes sense, it depends on several assumptions, including, as indicated, constant proportional tradeoffs and for instance the absence of discounting. Since the introduction of TTO [6] and its initial development, a considerable body of evidence has emerged showing that these assumptions do not always hold and, consequently, the way TTO is framed may influence the value given to health states [1, 7-9]. Therefore, differences in TTO values may arise from lack of standardisation of the TTO procedure.

However, variation may also occur when respondent samples included in studies differ in characteristics that are relevant to how respondents react to the type of question or to the actual valuation of health states. An important distinction that needs to made in this context is the difference between valuing one's own health state or a hypothetical health state. It has been shown repeatedly that direct TTO values (i.e., people valuing their own health state) are considerably higher than indirect TTO values (i.e., people valuing hypothetical health states) [10]. One reason for this is that own health state valuations, in contrast to those of hypothetical health states, may be influenced by coping [11, 12]. Here, we focus on indirect TTO valuations, as these are most often used as input for utility questionnaires like the HUI, EQ-5D and SF-6D and commonly used as source for national tariffs.

Next, we first highlight some elements of what we already know from literature about influences of person-related variables such as demographic and attitudinal characteristics on TTO responses, without trying to be exhaustive. Then, we argue that more systematic attention to the persons behind TTO responses is required for a better understanding of variations in their responses to TTO questions.

\section{What do we know?}

The influence of several demographic characteristics and attitudinal variables of respondents have been regularly reported in TTO studies, such as age, gender, marital status, having children, health status, education level, socioeconomic status, ethnicity, and religious beliefs. Below we highlight some of the findings regarding the influence of these variable on TTO values.

The evidence regarding the influence of age on TTO scores is mixed. Some studies observed no statistically significantly effects, although these typically used lower numbers of respondents or had less variance in age [5, 13]. Some large indirect TTO valuation studies investigated the relation between age and TTO. They found a non-linear relationship when using a fixed time frame of 10 years: generally valuations increase slowly up to the age of about 45 , fall slowly between age 45 and 70 , and then more sharply for older ages [14-16]. In terms of effect size, the influence of age mostly was relatively small [14].

For gender, most studies find no significant influence on TTO responses [13]. In studies that report a significant gender effect, women tend to give up less life years and thus value health states higher than men $[14,16,17]$.

Marital status, or living together with a partner [18], and having children [18], could influence TTO scores if these aspects would affect preferences for length over quality of life. Krol et al. [19] argued that two opposite effects may influence TTO scores in this context and provided evidence of their existence. On the one hand, the idea of leaving your loved ones behind can have a negative effect on the willingness to give up life years. On the other hand, people may not wish to be a burden to their loved ones due to the illness and, therefore, can be willing to give up more life years for a better quality of life in the years lived. In empirical work, this has mostly been investigated by looking at marital status and its association with TTO scores. The evidence here generally shows no relation with TTO scores (e.g., [20]), but sometimes a positive relation [16]. Krol et al. [19] indicate that the two opposite influences may, on average, cancel out. The scarce evidence on the effect of having children on TTO valuations suggests a negative effect on the willingness to give up life years and thus a positive influence on TTO scores, in particular among mothers [21].

Own health status could be expected to influence TTO scores as well, as it may induce forms of coping and adaptation and shifts of reference points. Studies investigating the relation between (self-reported) health and TTO scores find both insignificant [22] as well as significant effects [23]. Interestingly, Dolan [24] showed that, in addition to the potential influence the current health state of a respondent can have on TTO scores, negative thoughts respondents have about their future health, can also influence TTO scores. Respondents gave up more years in a TTO exercise to reduce such negative thoughts [24].

The evidence about the influence of socio-economic status on TTO responses is also mixed. For education level, studies report either no, positive or negative effects $[5,25]$. The same mixed results are observed for income [5, 26].

In addition, it is interesting to note that, without instructions, respondents generally do not take the financial consequences of ill health into account in TTO exercises, whereas mentioning loss of income because of illness explicitly only has a minor influence on TTO scores [22, 27-29]. 
Ethnicity generally does not appear to influence TTO scores [31], although some studies report a, typically small, effect [30].

Religion and religious beliefs could influence TTO scores not only through an influence on preferences for length over quality of life, but also due to the nature of the TTO exercise. One might expect some religious convictions to be connected with both a reluctance in engaging in a trade-off 'actively' shortening remaining life span to increase quality of life and attitudinal characteristics like fear of death [31]. The scarce evidence for religion suggests no statistically significant relation with TTO scores [32].

Related to the previous point, some studies explored the influence of attitudes towards life and death on TTO scores. This may be relevant since TTO questions often imply an early age of death and involve thinking about giving up life years. Significant associations have been found, for example, for beliefs regarding life after death [31, 33, 34], fear of death [31] and attitudes towards euthanasia [13, 31]. Respondents with a strong preference for staying alive 'at all costs' were reluctant to trade-off any years [35].

Expectations regarding length and future quality of life were also shown to play a role. For example, when subjective life expectancy exceeded the life duration specified in the TTO question, respondents were less willing to tradeoff years-and the higher subjective life expectancy, the lower the number of years traded-off [36, 37]. Expectations about future quality of life were found to be significant in one study [36].

These and some other person-related variables including cultural differences [38,39] and numeracy [4], have been explored in the TTO literature, but typically the evidence remains scarce and most influences were studied in isolation, often as 'by-catch' in valuation studies. In other words, there is a lack of studies investigating the influence of personrelated variables systematically.

\section{Why would we want to know?}

As we have highlighted above, the effect of person-related variables on TTO scores, or more precisely their association, tends to be small. Nevertheless, understanding these relations better, also in combination, is relevant for a number of reasons. First, knowledge of respondent characteristics that are influential for the outcomes of TTO studies may be helpful in sampling respondents. If TTO is used for generating nationally representative values for health states (often referred to as 'tariffs'), it may be important to sample respondents from the population according to such characteristics if and when relevant and influential. In addition, for comparability of results in time or across samples, it is useful to make the same selections of respondents and/or to correct for the same selection of potentially confounding variables. Moreover, better understanding of how different groups of respondents react to some of the defining features of TTO exercises, like the limited timespan and giving up life years, may help improve the design and further standardisation of the TTO method and other methods deriving health state valuations. Finally, the influence of respondent characteristics may also differ depending on the severity of the valued health state, which is directly relevant to TTO exercises. Demographic and/or attitudinal variables may exhibit a different influence when dealing with mild health states as compared to severe health states [22].

It is also relevant to investigate these respondent characteristics in combination. Let us consider age more closely, for example. Respondents of different ages may have different views about the importance of remaining length of life, the relevance of different dimensions of health for quality of life, or the relation between length and quality of life. Age may also be closely related to a number of other factors potentially influencing willingness to trade between length and quality of life. For instance, age may be associated with having (current or prior) experience with health problems, which may affect perceptions of how undesirable particular health states are. Age is also associated with role. Over time role functioning may shift from learning to being active in paid or unpaid activities, and include functioning as a partner, parent, or grandparent. And last but not least, age is related to health. If own health state influences TTO scores, then this effect, if not otherwise corrected for, may lead to an apparent association between age and TTO scores as well. Capturing all such elements under the umbrella of 'age' of course might not be adequate.

Moreover, while the influence of the separate variables on TTO scores may be small, the joint effect of several factors may be large. In that context, it is good to note that mostly, a large proportion of the observed variation in health state valuations or TTO scores remains unexplained, so far.

\section{What's next?}

We highlighted some of the current knowledge regarding the relation between TTO values of hypothetical health states and a variety of respondent characteristics. This evidence is largely a by-catch of valuation studies, fragmented and often mixed. We discussed several reasons why more systematic investigation of this relation is important for improving the design of TTO exercises and our understanding of the outcomes. Arnesen and Trommald [1] already concluded that two-thirds of the studies in their review did not present or discuss the influence of even basic respondent characteristics such as age and gender. The focus in TTO research thus seems to be primarily on mean sample results, and much 
less on the heterogeneity in values or associations between values and sample characteristics.

Considering the importance of reliable and valid estimates of quality of life for research and policy in the health care sector, and the prominent role of TTO in generating such values, more attention for standardisation in design and application of TTO and understanding of what drives TTO answers is warranted. This is also true for other health state valuation techniques such as the standard gamble and discrete choice experiments. Systematic analysis of the influence of respondent characteristics on the interpretation of TTO questions, the willingness to trade, and the number of years traded is an essential part of this process of standardisation. Improved knowledge regarding which factors influence TTO scores, can also inform sampling procedures.

It is clear that, to date, we insufficiently understand what influences TTO scores. Therefore, we argue that there is more that we need to know about the person behind the TTO.

\section{References}

1. Arnesen, T., Trommald, M.: Are QALYs based on time trade-off comparable? - a systematic review of TTO methodologies. Health Econ. 14(1), 39-53 (2005)

2. Attema, A.E., Brouwer, W.B.F.: The value of correcting values: influence and importance of correcting TTO scores for time preference. Value Health 13(8), 879-884 (2010)

3. Zarate, V., Kind, P., Chuang, L.: Hispanic valuation of the EQ-5D health states: a social value set for Latin Americans. Value Health 11(7), 1170-1177 (2008)

4. Woloshin, S., Schwartz, L.M., Moncur, M., Gabriel, S., Tosteson, A.N.: Assessing values for health: numeracy matters. Med. Decis. Mak. 21(5), 382-390 (2001)

5. Devlin, N.J., Tsuchiya, A., Buckingham, K., Tilling, C.: A uniform time trade off method for states better and worse than dead: feasibility study of the 'lead time' approach. Health Econ. 20(3), 348-361 (2011)

6. Torrance, G.W.: Toward a utility theory foundation for health status index models. Health Serv. Res. 11(4), 349-369 (1976)

7. Bleichrodt, H.: A new explanation for the difference between time trade-off utilities and standard gamble utilities. Health Econ. 11(5), 447-456 (2002)

8. van Osch, S.M., Wakker, P.P., van den Hout, W.B., Stiggelbout, A.M.: Correcting biases in standard gamble and time tradeoff utilities. Med. Decis. Mak. 24(5), 511-517 (2004)

9. Doctor, J.N., Bleichrodt, H., Lin, H.J.: Health utility bias: a systematic review and meta-analytic evaluation. Med. Decis. Mak. 30(1), 58-67 (2010)

10. de Wit, G.A., Busschbach, J.J.V., de Charro, F.T.: Sensitivity and perspective in the valuation of health status: whose values count? Health Econ. 9, 109-126 (2000)

11. Versteegh, M.M., Brouwer, W.B.F.: Patient and general public preferences for health outcomes: a call to reconsider current guidelines. Soc. Sci. Med. 165, 66-74 (2016)

12. Stolk, E.A., van Nooten, F.E.: Values for resource allocation should expose the adaptation process, not the outcome. Virtual Mentor. 7(2), pii: virtualmentor.2005.7.2.jdsc3-0502 (2005). https ://doi.org/10.1001/virtualmentor.2005.7.2.jdsc3-0502
13. Augestad, L.A., Rand-Hendriksen, K., Stavem, K., Kristiansen, I.S.: Time trade-off and attitudes toward euthanasia: implications of using 'death' as an anchor in health state valuation. Qual. Life Res. 22(4), 705-714 (2013)

14. Dolan, P., Gudex, C., Kind, P., Williams, A.: The time trade-off method: results from a general population study. Health Econ. 5(2), 141-154 (1996)

15. Dolan, P.: Effect of age on health state valuations. J Health Serv Res Policy. 5(1), 17-21 (2000)

16. Dolan, P., Roberts, J.: To what extent can we explain time tradeoff values from other information about respondents? Soc. Sci. Med. 54(6), 919-929 (2002)

17. Bernert, S., Fernández, A., Haro, J.M., König, H.H., Alonso, J., Vilagut, G., Sevilla-Dedieu, C., de Graaf, R., Matschinger, H., Heider, D., Angermeyer, M.C., ESEMeD/MHEDEA 2000 Investigators: Comparison of different valuation methods for population health status measured by the EQ-5D in three European countries. Value Health 12(5), 750-758 (2009)

18. Van Nooten, F.E., van Exel, N.J., Koolman, X., Brouwer, W.B.: Married with children the influence of significant others in TTO exercises. Health Qual Life Outcomes. 2(13), 94 (2015)

19. Krol, M., Attema, A.E., van Exel, J., Brouwer, W.: Altruistic preferences in time tradeoff: consideration of effects on others in health state valuations. Med. Decis. Mak. 36(2), 187-189 (2016)

20. Bhatnagar, V., Frosch, D.L., Tally, S.R., Hamori, C.J., Lenert, L., Kaplan, R.M.: Evaluation of an internet-based disease trajectory decision tool for prostate cancer screening. Value Health 12(1), 101-108 (2009)

21. van der Pol, M., Shiell, A.: Extrinsic goals and time tradeoff. Med. Decis. Mak. 27(4), 406-413 (2007)

22. Krol, M., Sendi, P., Brouwer, W.: Breaking the silence: exploring the potential effects of explicit instructions on incorporating income and leisure in TTO exercises. Value Health 12(1), 172180 (2009)

23. Ayalon, L., King-Kallimanis, B.L.: Trading years for perfect health: results from the health and retirement study. J Aging Health. 22(8), 1184-1197 (2010)

24. Dolan, P.: Thinking about it: thoughts about health and valuing QALYs. Health Econ. 20(12), 1407-1416 (2011)

25. Rowen, D., Brazier, J., Young, T., Gaugris, S., Craig, B.M., King, M.T., Velikova, G.: Deriving a preference-based measure for cancer using the EORTC QLQ-C30. Value Health 14(5), 721-731 (2011)

26. Guest, J.F., Sladkevicius, E., Gough, N., Linch, M., Grimer, R.: Utility values for advanced soft tissue sarcoma health states from the general public in the United Kingdom. Sarcoma. 2013, 863056 (2013)

27. Richardson, J., Peacock, S.J., Iezzi, A.: Do quality-adjusted life years take account of lost income? Evidence from an Australian survey. Eur J Health Econ. 10(1), 103-109 (2009)

28. Meltzer, D., Weckerle, C., Chang, L.M.: Do people consider financial effects in answering quality of life questions. Med. Decis. Mak. 19, 517 (1999)

29. Tilling, C., Krol, M., Tsuchiya, A., Brazier, J., van Exel, J., Brouwer, W.: Does the EQ-5D reflect lost earnings? Pharmacoeconomics. 30(1), 47-61 (2012)

30. Izadpanah, A., Sinno, H., Vorstenbosch, J., Lee, B.T., Lin, S.J.: Thigh laxity after massive weight loss: a utilities outcomes assessment. Ann. Plast. Surg. 71(3), 304-307 (2013)

31. van Nooten, F.E., van Exel, N.J., Eriksson, D., Brouwer, W.B.: "Back to the future": influence of beliefs regarding the future on TTO answers. Health Qual. Life Outcomes. 12(14), 4 (2016)

32. Essink-Bot, M.L., Stuifbergen, M.C., Meerding, W.J., Looman, C.W., Bonsel, G.J., VOTE group: Individual differences in the use of the response scale determine valuations of hypothetical 
health states: an empirical study. BMC Health Serv. Res. 27(7), $62(2007)$

33. Lamers, L.M., Stalmeier, P.F., Krabbe, P.F., Busschbach, J.J.: Inconsistencies in TTO and VAS Values for EQ-5D Health States. Med. Decis. Mak. 26(2), 173-181 (2006)

34. Rutten-van Mölken, M.P., Hoogendoorn, M., Lamers, L.M.: Holistic preferences for 1-year health profiles describing fluctuations in health: the case of chronic obstructive pulmonary disease. Pharmacoeconomics. 27(6), 465-477 (2009)

35. Kirsch, J., McGuire, A.: Establishing health state valuations for disease specific states: an example from heart disease. Health Econ. 9(2), 149-158 (2000)

36. van Nooten, F., Brouwer, W.: The influence of subjective expectations about length and quality of life on time trade-off answers. Health Econ. 13(8), 819-823 (2004)
37. van Nooten, F.E., Koolman, X., Brouwer, W.B.: The influence of subjective life expectancy on health state valuations using a 10 year TTO. Health Econ. 18(5), 549-558 (2009)

38. Johnson, J.A., Luo, N., Shaw, J.W., Kind, P., Coons, S.J.: Valuations of EQ-5D health states: are the United States and United Kingdom different? Med. Care 43(3), 221-228 (2005)

39. Knies, S., Evers, S.M., Candel, M.J., Severens, J.L., Ament, A.J., Knies, S., Evers, S.M., Candel, M.J., Severens, J.L., Ament, A.J.: Utilities of the EQ-5D: transferable or not? Pharmacoeconomics. 27(9), 767-779 (2009) 